\title{
Penetrating power characteristics of half-wavelength AC transmission in point-to-grid system
}

\author{
Hanqing LIANG ${ }^{1} \mathbb{D}$, Yadong LIU ${ }^{1}$, Lei WAN ${ }^{2}$, Gehao SHENG ${ }^{1}$, Xiuchen JIANG ${ }^{1}$
}

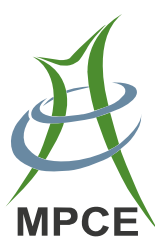

\begin{abstract}
With the concept of global energy interconnection being proposed, half-wavelength alternating current transmission (HWACT) technology becomes of more interest. HWACT lines can be adopted to establish a pointto-grid system, in which the penetrating power (PP) is produced between receiving terminals, having a significant effect on the power flow distribution. In order to investigate this phenomenon, the PP characteristics of the HWACT system are researched in this paper. First, the mathematical relationship between the transmission power and terminal bus voltages of a single HWACT line is derived using the equations of a distributed parameter model. The research indicates that the relationship between power and terminal voltages shows "reverse characteristics" opposite to those of regular short transmission lines. Then, the concept and
\end{abstract}

CrossCheck date: 16 January 2018

Received: 13 March 2017/Accepted: 16 January 2018/Published online: 23 March 2018

(c) The Author(s) 2018

$\triangle$ Hanqing LIANG

lianghanedu2016@163.com

Yadong LIU

liuyadong0916@163.com

Lei WAN

wleiwilson@163.com

Gehao SHENG

shenghe@sjtu.edu.cn

Xiuchen JIANG

xcjiang@sjtu.edu.cn

1 Department of Electrical Engineering, Shanghai Jiao Tong University, Shanghai 200240, China

2 Global Energy Interconnection Development and Cooperation Organization, Beijing 100031, China definition of PP in a point-to-grid system with two receiving terminals are proposed, and the corresponding relationship between PP and the terminal bus voltages is derived. Simulations are carried out to validate the theory under different conditions, so that the accuracy and adaptiveness of the theoretical analysis can be proved. In addition, the results demonstrate that selecting the location for a HWACT system has demanding requirements in order to control the value of PP.

Keywords Half-wavelength alternating current transmission (HWACT), Ultra-high voltage, Synchronous power grids, Penetrating power (PP)

\section{Introduction}

Half-wavelength alternating current transmission (HWACT) refers to a kind of AC transmission technology whose transmission distance is equal to a half of the wavelength under the fundamental frequency [1]. With the concept of global energy interconnection being proposed, HWACT technology becomes of more interest. In 2015, the State Grid Corporation of China (SGCC) launched a series of research projects on HWACT technology in order to seek alternatives for long-distance power transmission besides high voltage direct current (HVDC) transmission.

The equivalent reactance of a HWACT line is theoretically equal to zero. This can largely reduce the electrical distance between both ends of the line [2,3]. Because of this, HWACT is suitable for long-distance and high-capacity transmission [4-8]. For the traditional line, the resistance is far less than the reactance. Thus, the active power is strongly correlated with the phase angle of the 
node voltage whereas the reactive power is strongly related to the amplitude of the node voltage [9-14]. As the length of the line reaches the half-wavelength, the power-voltage characteristic becomes different. Under the assumption that the line is lossless, the voltage amplitudes of both ends are equal and the phase angle difference is 180 degrees for the HWACT line [1-3, 15]. This indicates that the terminal voltages are not related to the transmission power. However, further investigation is required to verify the correctness of this conclusion. The power-voltage characteristics also determine the overvoltage on the HWACT line. These could be serious when the transmission power is high $[3,16]$. If the power factor is equal to 1 , the overvoltage at the midpoint of the line is roughly equal to the ratio of the active power to the natural power $[1-3,17,18]$.

HWACT technology can also be used to enhance synchronous power grids. Reference [19] proposes the concept of building stereoscopic power grids using multiple HWACT lines. The HWACT lines share a common sending end and connect to different terminals in one power grid. The single point-double terminals (SPDT) system has been built in [19] and it is supposed to be the most typical system reflecting the networking capability of HWACT. The simulations demonstrate that the stability of the power grid can be enhanced effectively because the HWACT lines shorten the electrical distances between the terminals, changing the grids into a stereoscopic structure from a plane structure. Since it is a relatively new field, there are no more relevant references except [19]. Thus, some new features of the SPDT system are not yet completely clear. The power flow distribution on the HWACT lines is one of the key issues which need further study. The situation of the power flow distribution is quite complex in the SPDT system compared with that of a single line. Thus, it is necessary to study the power flow distribution characteristics of the SPDT system in depth.

Aiming at the above-mentioned problems, this paper derives and validates the power-voltage characteristics as well as the power distribution of the HWACT system. According to the equations of the distributed parameter model, the power-voltage characteristics of a single HWACT line are derived. The "reverse characteristics" of the HWACT line are found, opposite to those of regular short transmission lines. Then, the concept and definition of the penetrating power (PP) in a SPDT system is proposed. The corresponding relationship among PP, the terminal bus voltages and the supplied power can be obtained based on the principles and characteristics of PP. The research indicates that the relationship between PP and terminal voltages also shows "reverse characteristics". The simulations are carried out to validate the theoretical analysis under different conditions. The results indicate that demanding requirements should be made for the precision control of a HWACT system.

\section{Power-voltage characteristics of a single HWACT line}

A single AC transmission line is shown in Fig. 1, where $\dot{U}_{2}$ and $\dot{U}_{1}$ are the voltage phasors of both terminals, $\dot{I}_{2}$ and $\dot{I}_{1}$ are the current phasors of both terminals, $P$ and $Q$ are the active and reactive power from the terminal 2 to 1 , respectively.

The formula of the distributed parameter model can be written as [2]:

$\left[\begin{array}{c}\dot{U}_{1} \\ \dot{I}_{1}\end{array}\right]=\left[\begin{array}{cc}\cosh (\gamma l) & Z_{s} \sinh (\gamma l) \\ \frac{1}{Z_{s}} \sinh (\gamma l) & \cosh (\gamma l)\end{array}\right]\left[\begin{array}{c}\dot{U}_{2} \\ -\dot{I}_{2}\end{array}\right]$

where $\gamma$ is the transmission coefficient; $Z_{s}$ is the wave impedance; $l$ denotes the length of the line. $\gamma$ can be represented as $\alpha+\mathrm{j} \beta=\sqrt{z_{k} y_{k}}, Z_{s}$ can be represented as $\sqrt{z_{k} / y_{k}} \cdot z_{k}$ and $y_{k}$ represent the impedance, admittance per unit length, respectively. $z_{k}, y_{k}$ can be written as $r_{k}+\mathrm{j} x_{k}$, $g_{k}+\mathrm{j} b_{k}$, respectively. It can be found that $r_{k} \ll x_{k}$ and $g_{k} \ll b_{k}$.

Assuming that $\dot{U}_{2}=U_{2} \angle 0^{\circ}$ and $\dot{I}_{2}=I_{2} \angle-\varphi$, it can be found that:

$\dot{U}_{1}=\cosh (\gamma l) U_{2}-Z_{s} \sinh (\gamma l) \dot{I}_{2}$

$\left\{\begin{array}{l}P=U_{2} I_{2} \cos (\varphi) \\ Q=U_{2} I_{2} \sin (\varphi)\end{array}\right.$

$\dot{I}_{2}=\frac{P-\mathrm{jQ}}{U_{2}}$

According to (2)-(4), the equation of power-voltage characteristic can be represented as follows:

$\dot{U}_{1}=\cosh (\gamma l) U_{2}-Z_{s} \sinh (\gamma l) \frac{P-\mathrm{jQ}}{U_{2}}$

Then, the coefficient $\cosh (\gamma l)$ and $\sinh (\gamma l)$ can be expanded as:

$\left\{\begin{array}{l}\cosh (\gamma l)=\frac{1}{2}\left(\mathrm{e}^{\alpha l}+\mathrm{e}^{-\alpha l}\right) \cos (\beta l)+\mathrm{j} \frac{1}{2}\left(\mathrm{e}^{\alpha l}-\mathrm{e}^{-\alpha l}\right) \sin (\beta l) \\ \sinh (\gamma l)=\frac{1}{2}\left(\mathrm{e}^{\alpha l}-\mathrm{e}^{-\alpha l}\right) \cos (\beta l)+\mathrm{j} \frac{1}{2}\left(\mathrm{e}^{\alpha l}+\mathrm{e}^{-\alpha l}\right) \sin (\beta l)\end{array}\right.$

In order to analyze the effects of $\alpha$ and $\beta$, the derivation of $\mathrm{e}^{\alpha l} \cos (\beta l)$ and $\mathrm{e}^{\alpha l} \sin (\beta l)$ is shown as follows: 


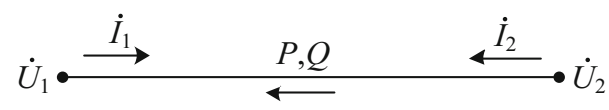

Fig. 1 Diagram of a single AC transmission line

$$
\left\{\begin{array}{l}
\frac{\mathrm{de}^{\alpha l} \cos (\beta l)}{\mathrm{d} l}=\mathrm{e}^{\alpha l}(\alpha \cos (\beta l)-\beta \sin (\beta l)) \\
\frac{\mathrm{de}^{\alpha l} \sin (\beta l)}{\mathrm{d} l}=\mathrm{e}^{\alpha l}(\alpha \sin (\beta l)+\beta \cos (\beta l))
\end{array}\right.
$$

According to (7), $\beta$ makes more contribution to parameter-growth if $\alpha / \beta<|\tan (\beta l)|<\beta / \alpha$. Since $\beta \gg \alpha$, the effect of $\alpha$ can be ignored, which is consistent with the hypothesis for the lossless line. On the other hand, if $|\tan (\beta l)|<\alpha / \beta$ or $|\tan (\beta l)|>\beta / \alpha$, the effect of $\alpha$ should not be ignored. As for the HWACT line, the value of $\beta l$ is equal to $\pi$, so (5) can be simplified to:

$\dot{U}_{1}=-\frac{1}{2}\left(\mathrm{e}^{\alpha l}+\mathrm{e}^{-\alpha l}\right) U_{2}+\frac{1}{2}\left(\mathrm{e}^{\alpha l}-\mathrm{e}^{-\alpha l}\right) Z_{s} \frac{P-\mathrm{jQ}}{U_{2}}$

Since the resistance is much smaller than the reactance per unit length, the imaginary part of the wave impedance $Z_{s}$ is very small. Thus, if the value of $U_{2}$ is determined, the real part of $\dot{U}_{1}$ is related to the active power whereas the imaginary part is related to the reactive power.

Taking the $1000 \mathrm{kV}$ ultra-high voltage (UHV) typical circuit as an example $\left(8 \times 500 \mathrm{~mm}^{2}\right.$ steel-cored aluminum wire), the calculated parameters are: $z_{k}=\left(8.01 \times 10^{-6}+\right.$ $\left.\mathrm{j} 2.631 \times 10^{-4}\right) \Omega / \mathrm{m}, \quad y_{k}=\mathrm{j} 4.3448 \times 10^{-9} \mathrm{~S} / \mathrm{m}$, $\alpha=1.6273 \times 10^{-8} \mathrm{~m}^{-1}, \beta=1.0693 \times 10^{-6} \mathrm{~m}^{-1}$. So the exact half-wavelength length can be calculated as $2938.3 \mathrm{~km}$. The voltage base $U_{B}$ is $1050 \mathrm{kV}$ and the power base $S_{B}$ is 100 MVA. So (8) can be modified as:

$\dot{U}_{1}=-1.00114 U_{2}+1.068 \times 10^{-3} \frac{P}{U_{2}}-\mathrm{j} 1.068 \times 10^{-3} \frac{Q}{U_{2}}$

Since the coefficients of $P, Q$ are small, the real part of (9) is much larger than the imaginary part when $P, Q$ are in a normal range. Thus, the amplitude of $\dot{U}_{1}$ has a higher correlation with the active power whereas the phase angle is more related to the reactive power. This feature shows "reverse characteristics" which is opposite to those of regular short transmission lines. Based on the previous derivation, it can be inferred that $Z_{s} \sinh (\gamma l)$ is the equivalent series impedance of $\pi$ circuit of the line. If $\beta l=\pi$, the equivalent reactance of $Z_{s} \sinh (\gamma l)$ is equal to zero in theory, so the coefficients of $P$ and $Q$ only reflect the effect of the equivalent resistance.

According to (9), the amplitude and phase angle variation of $\dot{U}_{1}$ is shown in Figs. 2 and 3. The value of $U_{2}$ is set at 1.0 p.u..

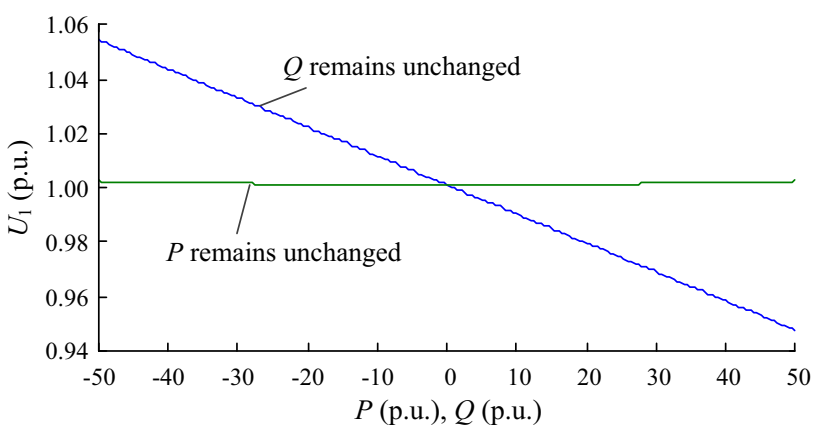

Fig. 2 Curves of terminal voltage amplitude varied with power for HWACT line

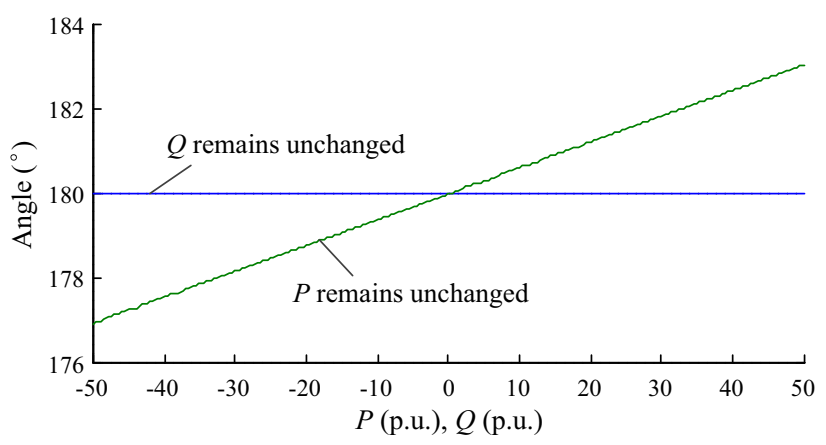

Fig. 3 Curves of terminal voltage phase angle varied with power for HWACT line

As shown in Figs. 2 and 3, if $P$ remains unchanged and $Q$ ranges from -50 to 50 p.u. ( $-5000-5000$ Mvar), the value of $U_{1}$ ranges from 1.0012 to 1.0026 p.u., and the maximum variation of the voltage amplitude is about 0.0014 p.u. In this case, the value of angle changes from $177^{\circ}$ to $183^{\circ}$, and the maximum variation of the phase angle is about $6^{\circ}$. According to the same figures, if $Q$ remains unchanged and $P$ changes in the same interval, the maximum variation of the voltage amplitude is approximately 0.1 p.u. (from 0.95 to 1.05 p.u.), while the phase angle remains $180^{\circ}$. The results verify the previous discussion to some extent. However, it also indicates that the inference from the hypothesis of the lossless line (the voltage amplitude of each terminal is equal and the phase angle difference is $180^{\circ}$ ) is not strictly correct. As for the HWACT line, the resistance cannot be ignored in some circumstances because it has impact on "reverse characteristics" opposite to those of regular short transmission lines.

\section{Power-voltage characteristics of SPDT system}

To derive the power-voltage characteristics of a SPDT system, two HWACT lines are adopted here to constitute the system, as shown in Fig. 4. Point $c$ connects to points 1 , 


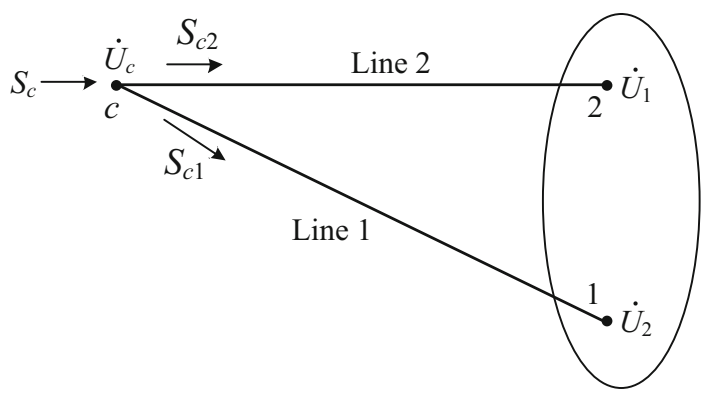

Fig. 4 Diagram of SPDT system

2 in a synchronous power grid by two HWACT lines, respectively.

$S_{c}$ represents the supplied output power at point $c . S_{c 1}$ and $S_{c 2}$ represent the transmission power from point $c$ to 1 , 2 , respectively. In this scenario, the electrical distance between the two separated points $(1,2)$ becomes shorter by using the HWACT line, so the mutual supporting capability can be improved. According to the analysis above, the PP which flows through the HWACT lines will be generated between point 1 and point 2 when there exists a voltage difference. The PP combines with $S_{c}$, leading to uneven distribution of the actual transmission power on each line. In this paper, PP is defined as:

$\Delta S_{p e} \triangleq \frac{1}{2}\left(S_{c 1}-S_{c 2}\right)$

It can be obtained as:

$\left\{\begin{array}{l}S_{c 1}=\frac{1}{2} S_{c}+\Delta S_{p e} \\ S_{c 2}=\frac{1}{2} S_{c}-\Delta S_{p e}\end{array}\right.$

According to (1), the relationship between voltage and current can be written as:

$\left[\begin{array}{c}\dot{I}_{c 1} \\ \dot{I}_{1}\end{array}\right]=\left[\begin{array}{cc}B^{-1} A & -B^{-1} \\ C-A B^{-1} A & A B^{-1}\end{array}\right]\left[\begin{array}{c}\dot{U}_{c} \\ \dot{U}_{1}\end{array}\right]$

where $\dot{I}_{c 1}$ is the current flowing from point $c$ to terminal 1; $A, B, \quad C$ denote $\cosh (\gamma l), Z_{s} \sinh (\gamma l)$, and $\sinh (\gamma l) / Z_{s}$, respectively.

The transmission power on Line 1 is:

$S_{c 1}=\dot{U}_{c} \dot{I}_{c 1}^{*}$

where the superscript * denotes the conjugate of the phasor. Combined with (12), (13) can be expanded as:

$$
\begin{aligned}
S_{c 1} & =\dot{U}_{c}\left(B^{-1} A \dot{U}_{c}-B^{-1} \dot{U}_{1}\right)^{*} \\
& =\left(B^{-1} A\right)^{*} U_{c}^{2}-\left(B^{-1}\right)^{*} \dot{U}_{c} \dot{U}_{1}^{*}
\end{aligned}
$$

Similarly, the transmission power on Line 2 is obtained as:
$S_{c 2}=\left(B^{-1} A\right)^{*} U_{c}^{2}-\left(B^{-1}\right)^{*} \dot{U}_{c} \dot{U}_{2}^{*}$

Thus, the PP can be derived as:

$\Delta S_{p e}=\frac{1}{2}\left(B^{-1}\right)^{*} \dot{U}_{c}\left(\dot{U}_{2}^{*}-\dot{U}_{1}^{*}\right)$

The supplied output power is written as:

$$
\begin{aligned}
S_{c} & =S_{c 1}+S_{c 2} \\
& =2\left(B^{-1} A\right)^{*} U_{c}^{2}-\left(B^{-1}\right)^{*} \dot{U}_{c}\left(\dot{U}_{2}^{*}+\dot{U}_{1}^{*}\right)
\end{aligned}
$$

As for the HWACT line, the value of $\beta l$ is equal to $\pi$, so the coefficients $A, B$ can be simplified as real numbers:

$$
\left\{\begin{array}{l}
A=\cosh (\gamma l)=-\frac{1}{2}\left(\mathrm{e}^{\alpha l}+\mathrm{e}^{-\alpha l}\right)=A^{*} \\
B=Z_{s} \sinh (\gamma l)=\frac{1}{2} Z_{S}\left(\mathrm{e}^{-\alpha l}-\mathrm{e}^{\alpha l}\right)=B^{*}
\end{array}\right.
$$

Combining (16)-(18), $\quad \dot{U}_{\mathrm{c}}$ is eliminated and the relationship among $S_{c}, \dot{U}_{1}, \dot{U}_{2}$ and $\Delta S_{p e}$ can be obtained as:

$S_{c}=8 A B\left|\frac{\Delta S_{p e}}{\dot{U}_{2}-\dot{U}_{1}}\right|^{2}-2 \Delta S_{p e}\left(\frac{\dot{U}_{2}+\dot{U}_{1}}{\dot{U}_{2}-\dot{U}_{1}}\right)^{*}$

1) Assuming that the terminal voltages are known. According to (19), $\Delta S_{p e}$ varies with $S_{c}$ nonlinearly. For example, the conditions are set as follows: $\dot{U}_{1}=0.95 \angle-3^{\circ}, \quad \dot{U}_{2}=1.0$, the power factor is 1 (reactive component $Q_{c}=0$ ). $P_{p e}$ and $Q_{p e}$ represent the active and reactive components of $\mathrm{PP}$, respectively. The curves of $P_{p e}, Q_{p e}$ varied with $P_{c}$ are presented in Fig. 5, which is produced according to (19). It can be seen that the PP varies with the supplied power non-linearly over a large range ( $-500-500$ p.u.) whereas the rate of change is small. Thus, the amplitude change of the PP is small when the supplied power ranges from -100 to 100 p.u., as is shown in the red frame.

2) Assuming that the supplied power is known. In the typical case of $S_{c}=0$, there is only PP flowing through the line. Thus, the equation can be obtained based on (18) and (19):

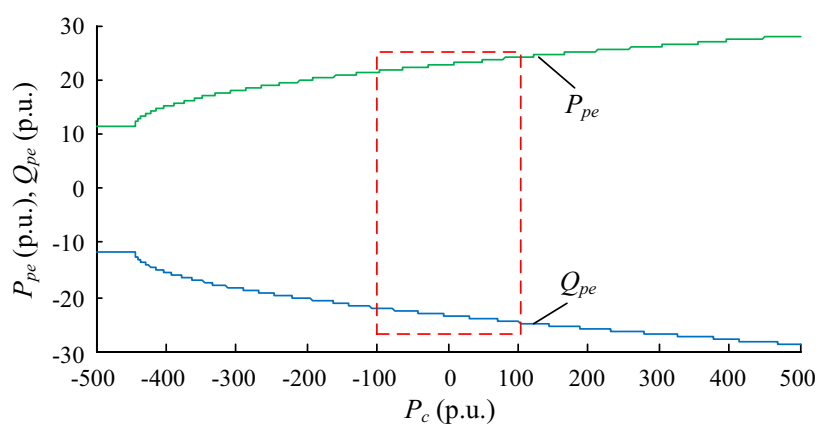

Fig. 5 Curves of PP varied with source power 
$S_{p c}=\frac{1}{Z_{s}^{*}\left(\mathrm{e}^{2 \alpha l}-\mathrm{e}^{-2 \alpha l}\right)}\left(U_{2}^{2}-U_{1}^{2}+\mathrm{j} 2 U_{1} U_{2} \sin \left(\varphi_{12}\right)\right)$

where $\varphi_{12}$ denotes the phase difference between terminals 1 and 2.

The active and reactive components of PP can be written as:

$$
\left\{\begin{array}{c}
P_{p e}=\frac{U_{2}^{2}-U_{1}^{2}}{\left|Z_{s}\right|\left(\mathrm{e}^{2 \alpha l}-\mathrm{e}^{-2 \alpha l}\right)} \\
Q_{p e}=\frac{2 U_{1} U_{2} \sin \left(\varphi_{12}\right)}{\left|Z_{s}\right|\left(\mathrm{e}^{2 \alpha l}-\mathrm{e}^{-2 \alpha l}\right)}
\end{array}\right.
$$

This shows that the active component of PP is only linearly related to the square error of voltage amplitude between the terminal points if there is no power being sent. On the other hand, the reactive component of $\mathrm{PP}$ is determined by both the voltage amplitude and the phase difference.

If $U_{2}>U_{1}$, then $P_{p e}>0$; that is, the active PP flows from the higher-voltage bus to the lower-voltage bus. On the other hand, if $\varphi_{12}>0$, then $Q_{p e}>0$. Therefore, the reactive PP flows from the smaller-phase angle bus to the larger-phase angle bus. Furthermore, the phasor diagrams of $U_{1}, U_{2}$ and $U_{c}$ can be obtained, which are presented in Fig. 6.

The above analysis indicates that the PP between the terminal points can be controlled by adjusting the voltage amplitude and phase angle of each terminal. In addition, the active and reactive power can be decoupled. Ideally, the value of PP should be controlled at zero to guarantee the maximum transmission power. The active PP can be controlled by arranging the amount of reactive power compensation at the terminal point properly. This contributes to adjusting the voltage amplitude. As for the reactive $\mathrm{PP}$, the phase angle can be controlled by adjusting the unit operation mode at the adjacent areas of terminal points.

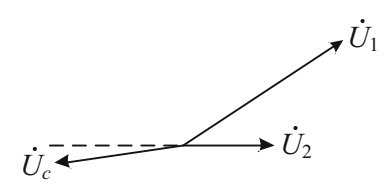

(a) $P_{p e}<0, Q_{p e}>0$

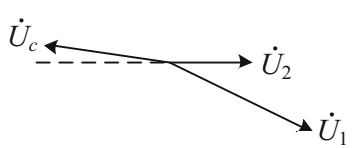

(c) $P_{p e}<0, Q_{p e}<0$

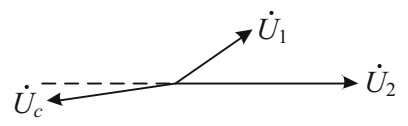

(b) $P_{p e}>0, Q_{p e}>0$

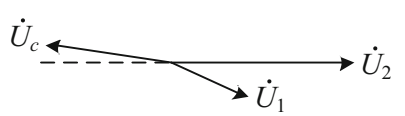

(d) $P_{p e}>0, Q_{p e}<0$
Fig. 6 Diagram of relationship among node voltage vectors in SPTD system

\section{Simulation verification}

In order to verify the theoretical analysis of the characteristics of a single HWACT line and SPDT system, PSD-BPA [20] is used to simulate the load flow distribution in a power grid. The HWACT line is modeled by $20 \pi$ sections connecting with each other [18]. The line length is set as $2938.3 \mathrm{~km}$.

\subsection{Simulation of single HWACT line}

As shown in Fig. 7, the simulation is carried out based on the case of planning UHV power grids of the SGCC for the year 2020. The HWACT line with voltage level of 1050 $\mathrm{kV}$ connecting the generator and a terminal bus is added to the original data. The location of the terminal bus is selected as Jingmen. Relevant parameters are presented in Fig. 7, where $S_{g}$ and $U_{g}$ represent the rated capacity and voltage of the sending unit, respectively, $S_{t}$ and $X_{k}$ are the rated capacity and leakage reactance of the transformer, respectively, $S_{b}=100 \mathrm{MVA}$ and $U_{b}=1050 \mathrm{kV}$ are the base capacity and base voltage of the system, respectively, $Z_{e q}$ and $Y_{e q}$ refer to the series impedance and the parallel admittance of each equivalent $\pi$ section, respectively.

The bus of Jingmen station is set as the balance node, whose voltage amplitude $U_{2}$ is set as 1.0 p.u., and the phase angle is $0^{\circ}$. To verify the "reverse characteristics" of the HWACT line, the theoretical and simulation results of the head-end voltage $U_{1}$ are compared under various $P$ and $Q$, which are shown in Table 1.

According to Table 1, if the reactive power remains unchanged and the active power is adjusted step-by-step, the terminal voltage mainly displays amplitude variation. However, if the reactive power is adjusted with the invariability of active power, the terminal voltage is changed mainly in phase angle. The amplitude errors between the theoretical and simulation results are in the $10^{-3}$ order of magnitude, and the phase angle errors are in the $10^{-2}$ order of magnitude. It can be seen that the theoretical and simulation results have high similarity. Thus, the correctness of the theory can be verified.

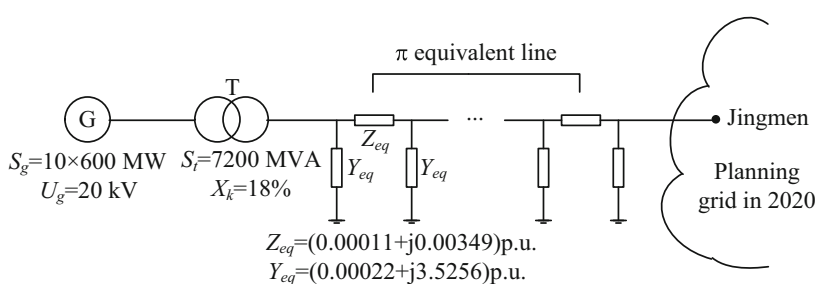

Fig. 7 Simulation case of single HWACT line connected to power grid 
Table 1 Comparison of theory and simulation results for single HWACT line

\begin{tabular}{lcccccc}
\hline$P$ (p.u.) & $Q$ (p.u.) & \multicolumn{2}{l}{ Theory result } & & \multicolumn{2}{c}{ Simulation result } \\
& & $U_{1}$ (p.u.) & $\varphi_{1}\left(^{\circ}\right)$ & & $U_{1}$ (p.u.) & $\varphi_{1}\left(^{\circ}\right)$ \\
\hline-50 & 0 & 1.0545 & 180.00 & & 1.0567 & 179.92 \\
-45 & 0 & 1.0492 & 180.00 & & 1.0512 & 179.92 \\
-40 & 0 & 1.0439 & 180.00 & & 1.0456 & 179.93 \\
-35 & 0 & 1.0385 & 180.00 & & 1.0401 & 179.94 \\
-30 & 0 & 1.0332 & 180.00 & & 1.0345 & 179.95 \\
-40 & -10 & 1.0439 & 179.41 & & 1.0454 & 179.32 \\
-40 & -5 & 1.0439 & 179.71 & & 1.0455 & 179.63 \\
-40 & 5 & 1.0439 & 180.29 & & 1.0458 & 180.24 \\
-40 & 10 & 1.0439 & 180.59 & & 1.0460 & 180.54 \\
\hline
\end{tabular}

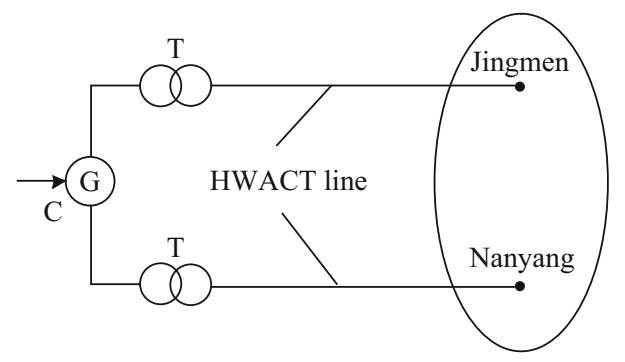

Fig. 8 Simulation solution for SPTD system

\subsection{SPTD system}

Based on the case of a single HWACT line, the second HWACT line is connected to the system. The location of the terminal is Nanyang, as shown in Fig. 8. In addition, the parameters of the second HWACT line are the same as the first line in the SPTD system. The relevant parameters are shown as: $Z_{s}=246.107 \Omega, \alpha=1.6273 \times 10^{-8} \mathrm{~m}^{-1}$. C refers to the location of the supplied output power.
Considering a simple situation where there is no power source, the value of PP can be calculated based on (20). The comparison of the theoretical and simulation results for PP is presented in Table 2. $U_{2}$ and $U_{1}$ are the voltage of Jingmen, Nanyang station, respectively. $\varphi_{12}$ represents the phase angle difference. $P_{c r}$ and $Q_{c r}$ represent the active and reactive $\mathrm{PP}$, respectively.

As can be seen in Table 2, the PP will change over a wide range if the value of the terminal voltages is adjusted. The simulation results have shown satisfactory agreement with theory. The maximum errors of the active and reactive PP are 0.92 p.u. (92 MW), 0.21 p.u. (21 Mvar), respectively.

The relation between the active PP and terminal voltages is presented in Fig. 9. It can be inferred that the active PP varies linearly with $U_{2}^{2}-U_{1}^{2}$. This is identical to the theoretical results. The relation between the reactive PP and $\varphi_{12}$ is shown in Fig. 10. The phase angle ranges from $-3.8^{\circ}$ to $-0.8^{\circ}$. Thus the simulation and theory results have high similarity. Since the value of $\varphi_{12}$ is small, $\sin \left(\varphi_{12}\right) \approx \varphi_{12}$. Thus, the reactive PP is strongly correlated to the phase angle difference between the terminal buses.

According to Figs. 9 and 10, the PP shows great variation with the voltage amplitude and the phase angle at terminals. That is, a little voltage difference can lead to a large PP. It can be seen that the active power is more correlated with the amplitude of the bus voltage whereas the reactive power is more related to the phase angle.

Since the supplied power may not be zero in reality, simulations are also performed with different supplied power, as shown in Fig. 11. It can be seen that PP is mainly related to the terminal voltages, and the variation ratio with $S_{c}$ is very small. In Fig. 11, with the variation scale 100 p.u. of $S_{c}$, the variation scale of PP is less than 3 p.u.. The conclusion is consistent with the theoretical curve of Fig. 5.

Table 2 Comparison of theory and simulation results for SPTD system

\begin{tabular}{|c|c|c|c|c|c|c|}
\hline \multirow[t]{2}{*}{$U_{2}$ (p.u.) } & \multirow[t]{2}{*}{$U_{1}$ (p.u.) } & \multirow[t]{2}{*}{$\varphi_{12}\left({ }^{\circ}\right)$} & \multicolumn{2}{|c|}{ Theory result } & \multicolumn{2}{|c|}{ Simulation result } \\
\hline & & & $P_{c r}$ (p.u.) & $Q_{c r}$ (p.u.) & $P_{c r}$ (p.u.) & $Q_{c r}$ (p.u.) \\
\hline 1.0124 & 0.9960 & -3.216 & 7.70 & -26.46 & 8.49 & -26.37 \\
\hline 1.0129 & 1.0023 & -3.382 & 5.00 & -28.02 & 5.82 & -28.00 \\
\hline 1.0086 & 1.0052 & -3.626 & 1.60 & -29.99 & 2.42 & -30.09 \\
\hline 1.0058 & 1.0064 & -3.761 & -0.24 & -31.06 & 0.66 & -31.22 \\
\hline 1.0032 & 1.0074 & -3.896 & -1.98 & -32.12 & -1.06 & -32.33 \\
\hline 1.0107 & 0.9993 & -2.789 & 5.34 & -22.99 & 6.02 & -22.94 \\
\hline 1.0078 & 1.0045 & -2.084 & 1.54 & -17.22 & 2.04 & -17.24 \\
\hline 1.0059 & 1.0076 & -1.603 & -0.80 & -13.26 & -0.42 & -13.33 \\
\hline 1.0030 & 1.0134 & -0.777 & -4.91 & -6.45 & -4.72 & -6.61 \\
\hline
\end{tabular}




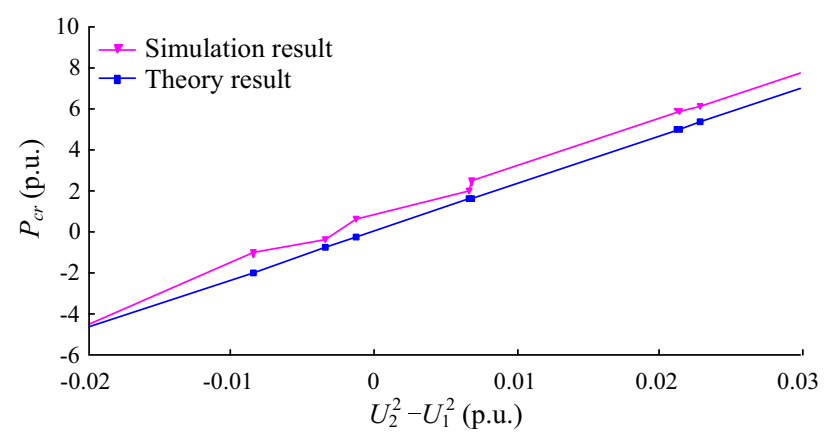

Fig. 9 Active PP varied with amplitude of terminal voltages

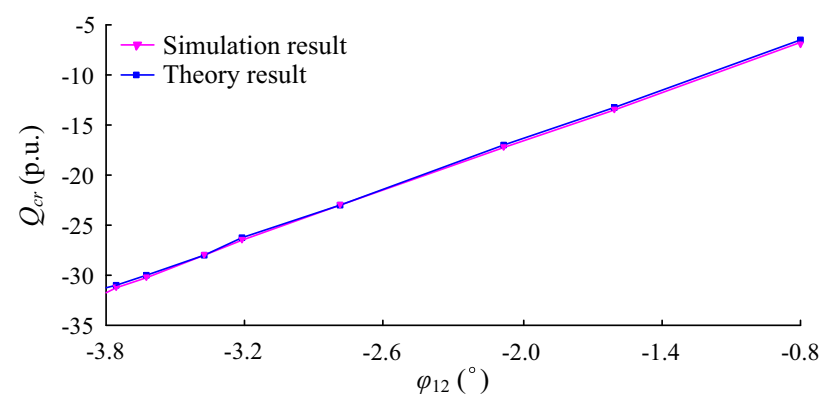

Fig. 10 Reactive PP varied with phase angle of terminal voltages

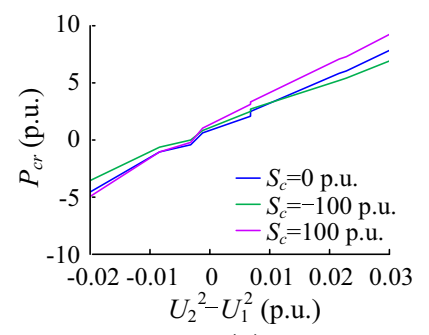

(a)

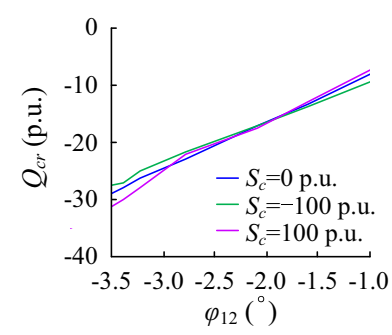

(b)
Fig. 11 PP varied with terminal voltages in different supplied powers

To further verify the proposed theory, more simulations are carried out under different conditions, as shown in Figs. 12 and 13. The characteristics of PP are validated with different voltage grades and propagation coefficients. Thus, the accuracy and adaptiveness of the theoretical analysis can be proved.

\section{Conclusion}

In this paper, the "reverse characteristics" of the HWACT line and the concept of PP have been proposed.

1) The HWACT line shows "reverse characteristics" opposite to those of regular short transmission lines. The active power is more correlated with the amplitude of the bus voltage whereas the reactive power is more related to

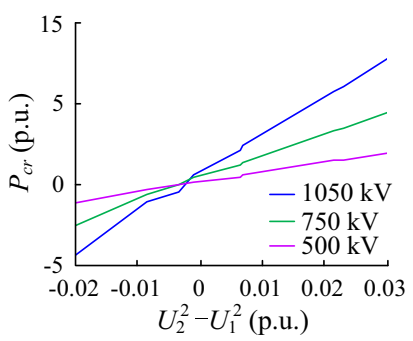

(a)

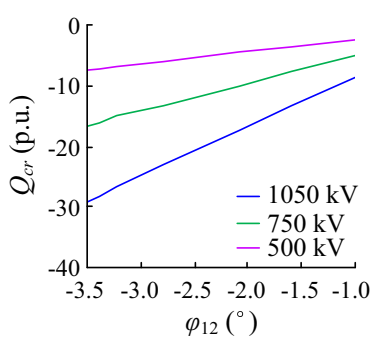

(b)
Fig. 12 PP varied with terminal voltages in different voltage grades

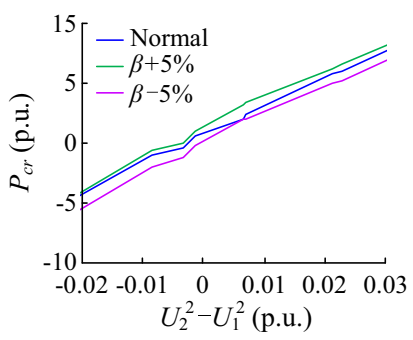

(a)

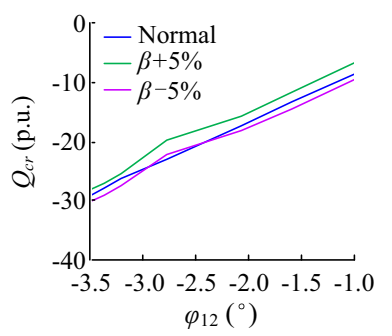

(b)
Fig. 13 PP varied with terminal voltages in different propagation coefficients

the phase angle. From this perspective, HWACT is similar to DC transmission. Thus, the resistance should not be ignored in the calculation and analysis of the HWACT line.

2) The power flow distribution of the SPTD system is analyzed. This helps reveal the characteristics of the PP on the HWACT line. PP is much more related to terminal voltages than the supplied power. The active PP is linearly related to the square error of amplitudes of the terminal voltages whereas the reactive PP is highly determined by the phase angle difference of the terminal voltages. Little difference of the voltage amplitude and phase angle will lead to a large PP. Thus we can infer that different control methods should be adopted in the HWACT system. In addition, the accuracy and reliability of the control is demanding, and the terminal locations of the HWACT lines need to be selected carefully.

3) This paper provides a preliminary study on the related problems of the HWACT system. This should be helpful for the application of HWACT technology. In future work, control methods for the HWACT system as well as the characteristics of the mixed HWACT system (such as the grid-to-grid system in which two independent power grids are connected by several HWACT lines) will be further researched.

Acknowledgements This work was supported by National Natural Science Foundation of China (No. 51307109) and State Grid Corporation of China (No. XT71-16-001). The authors would also thank for the technique support of China Electric Power Research Institute. 
Open Access This article is distributed under the terms of the Creative Commons Attribution 4.0 International License (http:// creativecommons.org/licenses/by/4.0/), which permits unrestricted use, distribution, and reproduction in any medium, provided you give appropriate credit to the original author(s) and the source, provide a link to the Creative Commons license, and indicate if changes were made.

\section{References}

[1] Hubert FJ, Gent MR (1965) Half-wavelength power transmission lines. IEEE Trans Power Appar Syst 84(10):965-974

[2] Prabhakara FS, Parthasarathy K, Rao HR (1969) Analysis of natural half-wave-length power transmission lines. IEEE Trans Power Appar Syst PAS-88(12):1787-1794

[3] Iliceto F, Cinieri E (1988) Analysis of half-wavelength transmission lines with simulation of corona losses. IEEE Trans Power Deliv 3(4):2081-2091

[4] Santos MLD, Jardini JA, Casolari RP et al (2014) Power transmission over long distances: economic comparison between HVDC and half-wavelength line. IEEE Trans Power Deliv 29(2):502-509

[5] Dias R, Lima A, Portela C et al (2011) Extra long-distance bulk power transmission. IEEE Trans Power Deliv 26(3):1440-1448

[6] Liu S, Wang X, Ning L et al (2017) Integrating offshore wind power via fractional frequency transmission system. IEEE Trans Power Deliv 32(3):1253-1261

[7] Aredes M, Dias R (2012) FACTS for tapping and power flow control in half-wavelength transmission lines. IEEE Trans Industrial Electron 59(10):3669-3679

[8] Portela C, Aredes M (2003) Very long distance transmission. http://www.dsce.fee.unicamp.br/ cristina/palestras/FURNAS/ documentos/AB02novosibart1Bcop2cpc.pdf. Accessed 10 January 2003

[9] Carvalho PM, Correia PF, Ferreira LA (2008) Distributed reactive power generation control for voltage rise mitigation in distribution networks. IEEE Trans Power Syst 23(2):766-772

[10] Moger T, Dhadbanjan T (2015) A novel index for identification of weak nodes for reactive compensation to improve voltage stability. IET Gener, Transm \& Distrib 9(14):1826-1834

[11] Kundur P (1994) Power system stability and control. McGrawhill, New York

[12] Han Y, Li H, Shen P et al (2017) Review of active and reactive power sharing strategies in hierarchical controlled microgrids. IEEE Trans Power Electron 32(3):2427-2451

[13] Li Y, Mai R, Lu L et al (2017) Active and reactive currents decomposition-based control of angle and magnitude of current for a parallel multiinverter IPT system. IEEE Trans Power Electron 32(2):1602-1614

[14] Renedo J, Garci A, Rouco L (2016) Active power control strategies for transient stability enhancement of AC/DC grids with VSC-HVDC multi-terminal systems. IEEE Trans Power Syst 31(6):4595-4604

[15] Zhang L, Zou L, Lu X et al (2012) Power frequency temporary overvoltage on half-wave length AC transmission lines. High Volt Eng 38(10):2728-2734

[16] Gatta FM, Iliceto F (1992) Analysis of some operation problems of half-wave length power transmission lines. In: Proceedings of
African electrical technology conference. Royal Swazi Sun, Swaziland, 22-24 September 1992, pp 59-64

[17] Esmeraldo PCV, Gabaglia CPR, Aleksandrov GN et al (1999) A proposed design for the new Furnas $500 \mathrm{kV}$ transmission linesthe high surge impedance loading line. IEEE Trans Power Deliv 14(1):278-286

[18] Qin X, Zhang Z, Xu Z et al (2011) Study on the steady state characteristic and transient stability of UHV AC half-wavelength transmission system based on quasi-steady model. Proc CSEE 31(31):66-76

[19] Liang XM, Zhang YT, Qin XH (2016) Study on the stereoscopic power grid construction based on UHV AC half-wave-length transmission technology. Power Syst Technol 40(11):3415-3419

[20] Tang Y, Bo GQ, Hou JX (2007) PSD-BPA transient stability program user's manual. China Electric Power Research Institute, Beijing

Hanqing LIANG received the B.S. degree in electrical engineering from North China Electric Power University, Hebei, China, in 2015. $\mathrm{He}$ is currently pursuing the Ph.D. degree in electrical engineering at Shanghai Jiaotong University, Shanghai, China. His main research interests include detecting fault location and diagnosis of power equipment.

Yadong LIU received his M.D. and Ph.D. degrees in electrical engineering from Shanghai Jiao Tong University, Shanghai, China, in 2008 and 2012 respectively. Currently, he is a lecturer in School of Electronic Information and Electrical Engineering at Shanghai Jiao Tong University. His main research interests include detecting fault location and diagnosis of power equipment.

Lei WAN received his B.E. degree from Tsinghua University, Beijing, China, in 2006 and M.D. degree from China Electric Power Research Institute, Beijing, China, in 2009, both in electrical engineering. Currently he is a senior engineer at Global Energy Interconnection Development and Cooperation Organization (GEIDCO), Beijing, China. His main research interests include half-wavelength AC transmission, high voltage direct current transmission and power system planning.

Gehao SHENG received his B.E. and Ph.D. degrees from Huazhong University of Science and Technology, Wuhan, China, in 1996 and 2003, respectively. Currently he is an associate professor in electrical engineering at Shanghai Jiao Tong University, Shanghai, China. His main research interests include control theory and its applications to automatic voltage control in power systems.

Xiuchen JIANG received his M.D. degree in electrical engineering from Tsinghua University, Beijing, China, in 1992, and his Ph.D. degree in electrical engineering from Shanghai Jiao Tong University in 2001. Currently, he is a full professor at Shanghai Jiao Tong University, acting as the director of Electrical Power Department at Shanghai Jiao Tong University since 2002. His main research interests include electrical measuring technology and electrical apparatus automation. 